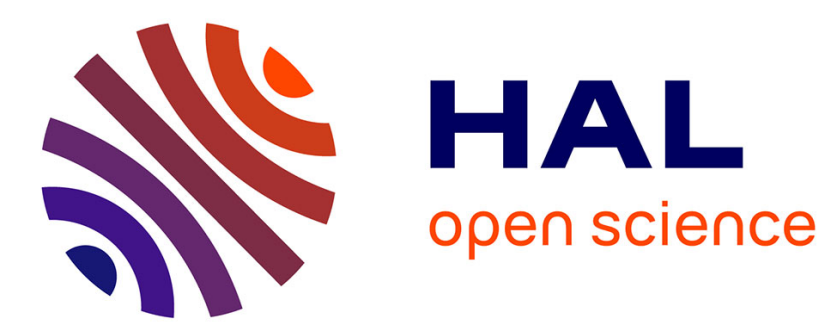

\title{
Capturing Chromosome Structural Properties From Their Spatial and Temporal Fluctuations
}

\author{
Aurélien Bancaud, Olivier Gadal, Kerstin Bystricky
}

\section{To cite this version:}

Aurélien Bancaud, Olivier Gadal, Kerstin Bystricky. Capturing Chromosome Structural Properties From Their Spatial and Temporal Fluctuations. Epigenetics and Systems Biology, 2017, 9780128030752. hal-01682736

\section{HAL Id: hal-01682736 \\ https://hal.science/hal-01682736}

Submitted on 12 Jan 2018

HAL is a multi-disciplinary open access archive for the deposit and dissemination of scientific research documents, whether they are published or not. The documents may come from teaching and research institutions in France or abroad, or from public or private research centers.
L'archive ouverte pluridisciplinaire HAL, est destinée au dépôt et à la diffusion de documents scientifiques de niveau recherche, publiés ou non, émanant des établissements d'enseignement et de recherche français ou étrangers, des laboratoires publics ou privés. 


\section{Capturing chromosome structural properties from their spatial and temporal fluctuations}

Aurélien Bancaud ${ }^{1}$, Olivier Gadal ${ }^{2}$, Kerstin Bystricky²

1 LAAS-CNRS, Université de Toulouse, CNRS, Toulouse, France

2 Laboratoire de Biologie Moléculaire Eucaryote (LBME); Centre de Biologie Intégrative (CBI); Université de Toulouse; CNRS; 118 route de Narbonne, F-31062 Toulouse, France

Running title: Live cell microscopy to determine chromosome structure

Keywords: chromosome organization, yeast genome, live cell imaging, chromatin/DNA labeling, chromosome/DNA dynamics, high throughput 3D imaging, chromosome biophysics, chromosome compaction, chromosome flexibility 


\section{Content}

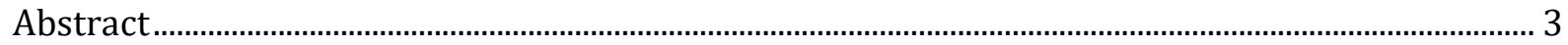

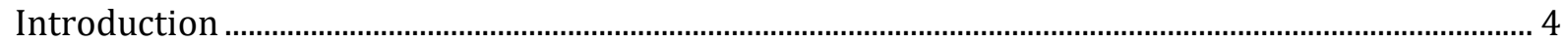

1- Chromosome labeling technologies ………………........................................................................... 5

2- Spatial dynamics: Real time imaging and tracking of single chromosome loci ................................ 7

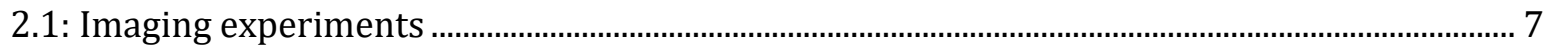

2.2: A few facts on the signal to noise ratio in single particle experiments ........................................ 8

2.3: Tracking and trajectory reconstruction ........................................................................................ 9

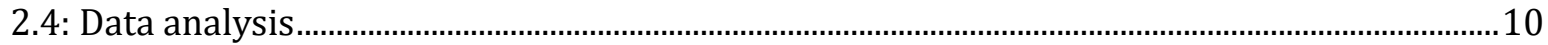

3: Mapping chromosome conformational dynamics by single/multi loci positioning ......................12

3.1: Single locus localization in the nucleus in a large population of cells ......................................12

3.2: Multi-locus localization for chromosome conformation analysis ...............................................13

3.2: Real-time tracking of multiple loci ……...........................................................................................14

4: Outlook - future developments.......................................................................................................15

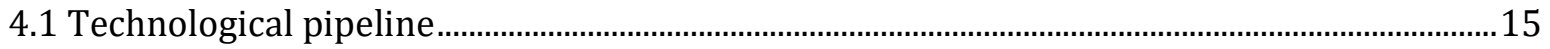

4.2: Chromosome structure and dynamics and their link to genome transactions.........................16

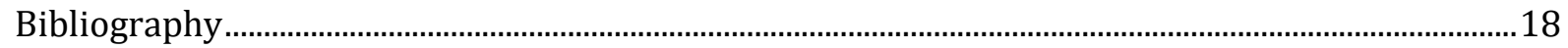

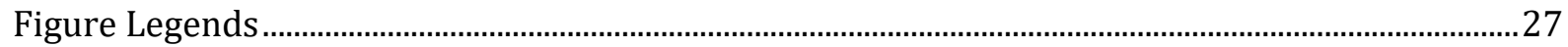

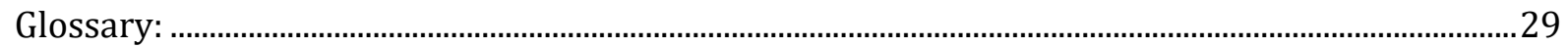

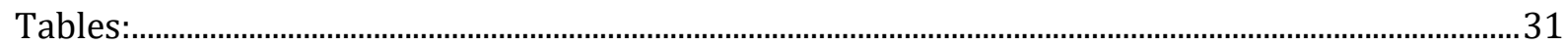




\begin{abstract}
Chromosome folding and nuclear organization are influenced by cellular processes while regulating genome functions. Intense efforts based on interdisciplinary research at the forefront of physics, mathematics, molecular and cellular biology and biochemistry have contributed to a better understanding of the physical properties and mechanisms of chromosome conformation. In this chapter, we focus on technologies for capturing chromosome spatial and temporal fluctuations based on the realization that chromatin conformation is highly dynamic at every length and time scale. We describe state of the art chromatin labeling technologies, focusing on optimizing yield of signal-to-noise ratio, high-throughput automated imaging techniques, and physical modeling. Systems biology approaches to map chromosome conformation using single or multiple labeled loci are illustrated, and implications of chromosome structural fluctuations on biological functions are discussed.
\end{abstract}




\section{Introduction}

20 years ago the first whole genome, the DNA sequence of the baker's yeast Saccharomyces cerevisiae, was sequenced (1). Many others followed, from bacteria to vertebrates and plants, whose genomes comprise between one million to several dozens of billion nucleotides. These genomes were unveiled close to completeness opening the door to pan-genomic comparisons and, although debated, personalized medical diagnostics (2-6). This one-dimensional (1D) information is precious, but understanding the intricacies of genome function requires exploring additional levels. One of them, the Epigenome, a refined 'dress-code' of the DNA fiber in eucaryotes, brought us one step closer to comprehending how nuclear functions are regulated. How the epigenome itself is organized and operational requires of us to place it into a 3D context. Spatiotemporal dynamics of the genome, or 4D Nucleome, have been shown to respond to endogenous and exogenous cues: physiological (a striking example is that in photoreceptor rod cells heterochromatin zones are inverted to accommodate nocturnal vision (7)), hormonal (hormone stimulation alters general chromatin compaction in human cancer cells (8)) or metabolic (yeast nuclear size and gene expression reorganizes when changing the carbon source (9)) to cite just a few examples. 3D position or chromatin fiber conformation further influence cellular functions, notably gene expression, recombination and repair are less efficient near the nuclear periphery where compaction levels are presumably greater than elsewhere in the nucleus (10-13). Transcriptional silencing is thought to largely depend on formation of denser structures as seen for entire genomes (spermatozoa or erythrocytes (14, 15)), whole chromosomes (the Barr body representing X-inactivation in mammals (16)), chromosome domains (chromocenters in mice and plants $(17,18)$ ) or specific loci (mating type loci in yeast $(19,20)$ or imprinted loci in mammals $(21,22))$. Numerous examples exist where changes in 4D nucleome features have been correlated with dedifferentiation, genome instability and disease (23-26).

Therefore, elucidation of physical mechanisms governing chromosome folding and their interplay with genome functions are the subject of intense research (27). High resolution chromosome conformation capture (3C)-based methods (see (28) for a recent review), which become more and more popular for exploring 3D organization of genomes, have been instrumental in determining the folding of chromosomes at length scales spanning $10 \mathrm{~kb}$ up to Gb. They provide contact frequency maps that are indicative of the occurrence at which sequences are ligated together after cross-linking (29). High frequencies correlate strongly with spatial proximity but are prone to fixation artifacts which can be normalized to some extent (3032), and others that call for additional information usually obtained with direct imaging 
observation (33-35). These imaging studies have been essentially based on physical distance measurements between chromosome loci separated by given genomic distances, and they provided some of the first insights on the folding principles of chromosomes at length scales from $100 \mathrm{~nm}$ to a few $\mu \mathrm{m}((36,37)$, see $(38)$ for a review).

Chromatin loci also exhibit fast spatio-temporal dynamics, which have been extensively documented since the development of labeling methods based on bacterial operator systems by the Murray lab in 1997 (39). Chromosomes undergo random fluctuations but also infrequent directional movements (40) associated with transcription activation (41). Regulation of chromosome dynamics is influenced by the spatial organization of genes during transcription (42-45) and DNA repair (46-49). At larger scales chromosome reorganization events detected during development (50), could be correlated to transcription activation (51). More recently, approaches, in particular displacement correlation spectroscopy based on time-resolved image correlation analysis (52) to map chromatin dynamics simultaneously across the whole nucleus have been developed to infer chromosome large-scale displacements. The modern view is that chromosomes are increasingly dynamic at every length scale from the molecular level - where the rapid turnover of chromatin associated proteins has been observed long ago by photobleaching techniques (53) - to the level of chromosome territories. However the physical mechanisms that govern these fluctuations remain elusive at any length scale, and raise questions about the exact contribution of active forces (54), which may bring chromatin to a farfrom-equilibrium state.

In this review, we focus on technologies for chromosome loci analysis, and discuss the insights on chromosome properties in relation to their biological functions that can be gained applying them. We will first overview methods to label single or multiple chromosome loci in living cells, then discuss the techniques to perform particle tracking and high throughput localization imaging, systems biology approaches for automated detection and statistical analysis and the models to analyze the data. We finally discuss whether or not chromatin motion and quantitative positional analysis are relevant to investigate chromosome properties and propose some experiments to gain confidence and/or refine the structural insights gained with models.

\section{1- Chromosome labeling technologies}

Chromatin dynamics can be analyzed at multiple scales in space and time using different fluorescence-based imaging methods. At great length scales, the movements of genome domains 
were followed by confocal microscopy using fluorescently tagged histones $(55,56)$, generally performed after partial photo-bleaching or photo-activation. The minimal area that can be studied with this approach is defined by the size of the laser spot used to photo-bleach or photoconvert fluorescent histones (57), which encompasses several Mb to Gb of chromatin. Live cell tracking of telomeres over several minutes corroborated the notion of self-contained chromosome domains in yeast (58) and metazoan cells (59). 3C-based technologies revealed the existence of topologically associated domains (TADs) of smaller dimensions in the range 0.1 to $10 \mathrm{Mb}(60)$, within which DNA-DNA interactions are more frequent as compared to the bulk of the genome. Elucidating their origin and structural bases requires new microscopy techniques, including collections of specific DNA probes to label target region (61), which are so far limited to analysis in fixed cells. At smaller length scales, visualization of chromosome loci can be performed without sequence specificity by incorporating injected or transfected fluorescent nucleotides during replication (62), or indirectly, by expressing photo-convertible fluorescent histones (63). The former technique achieves excellent signal to noise ratio (SNR, see more below) when labeling "large" loci of $\sim 50 \mathrm{~kb}$ (64). whereas the latter permits analysis of trajectories even of single nucleosomes. Visualization of DNA at specific genomic loci requires labeling techniques that create a fluorescent focus detectable above background levels. Several systems useful for live cell imaging have been developed (Table 1), for a review see ref. (65). FROS (fluorescent repressor operator system, (66)) is based on insertion of numerous repeats of bacterial operator sequences (150-250bp per unit) to which fluorescent repressor fusion proteins bind. The FROS system includes TetR/tetO (66), LacI/lacO (67), $\lambda \mathrm{cl} / \lambda \mathrm{Op}$ (68). Although widely used and very powerful, genome biology may be affected by FROS insertion, and biological consequences should be tested before and after insertion (69). FROS insertion can promote heterochromatin formation at a nearby silenced locus (70), and block DNA or RNA polymerase progression. Operator repeats should therefore be inserted as far as possible from regulatory elements, but still close enough to be considered part of the tagged and tracked locus to reduce possible side effects. This can be $100-800 \mathrm{bp}$ in $3^{\prime}$ and $5^{\prime}$ of transcribed genes or several kb when studying telomeres.

Taking advantage of protein oligomerization rather than DNA sequence repeats, Suntag (71). based on antibody recognition of amplified protein segments, and ANCHOR (ParB/INT), based on bacterial partitioning complexes ((72); Germier et al. submitted, Mariame et al. unpublished) are less invasive, yet stable new labeling techniques available for use in yeast and most metazoan cells. Unfortunately, quantitative comparison of the different labelling technologies in terms of SNR and motion is largely missing. Different size (number of repeats) of lacO arrays have been used to distinguish two DNA loci in yeast (for example $(73,74)$ ), but only 
qualitatively. Several groups have implemented reduced size operator arrays and assessed their suitability for detecting position or even live cell tracking, yet most of these analyses based on SNR are not published. A very careful analysis of the impact of photo-bleaching on yeast cell viability with respect to fluorescent intensity and imaging quality was performed by the Sedat lab using the OMX microscope (75). They demonstrated that $1 \%$ of usual excitation intensity can suffice to track loci using the OMX and applying denoising algorithms. The ANCHOR1 and ANCHOR 2 (ParB1 - ParB2/INT) systems have a lower SNR than the lac and tet FROS, on average, in yeast, but spots can be tracked as efficiently using a spinning-disk (Nipkow-disk) confocal system. Improved resolution and SNR for point-like signals is achieved because only a small fraction of the sample is visible at any given instant through a series of pin-holes on a rapidly rotating disk. The ANCHOR3 (Germier et al. submitted, Audibert et al. unpublished) system offers greatly improved SNR in both yeast and mammalian cells.

The use of CRISPR (Clustered Regularly Interspaced Short Palindromic Repeats) and inactivated or dead CRISPR-associated (dCas9) protein fused to fluorescent proteins is very promising to label multiple sites transiently. Sites are specifically targeted by short guide RNAs complementary to genomic CRISPR sequences $(76,77)$ or TALE $(78,79)$. Here also, little quantitative data is available. The first study (76) showed that dCas9-GFP labeled, naturally occurring repetitive regions colocalize with corresponding FISH probes in fixed cells. They quantified movement of telomeres visualized by dCas9-GFP targeted to telomere specific guide RNAs and compared it to the one recorded using the telomere protein TRF1 fused to GFP. The detected motion was heterogeneous in both cases. Fluorescence intensity relative to the determined diffusion coefficient was plotted to exclude any microscopy artifacts. Combining dCas9 labeling with the MS2 RNA-tagging system improves the capabilities for multicolor labeling but the SNR is still limited (80). Recently, the Pederson group demonstrated that several dCas9 from different bacteria allowed visualization of multiple spots which could also be tracked, but quantitation here also needs optimization: intensity or efficiency of the orthologous systems with respect to other labeling techniques have yet to be estimated (77).

\section{2- Spatial dynamics: Real time imaging and tracking of single chromosome loci}

\section{1: Imaging experiments}

Chromosome loci tracking is carried out with different fluorescence microscopes. Citing only the main setups including (multiphoton) confocal, spinning disk, wide-field, or totalinternal reflection microscopes (see refs. (81-83) as reviews). Depending on the application, the 
microscopy technique is chosen to track objects in 2D or 3D with optimal temporal resolutions; the choice of the light source as well as the photo-detector (cameras or diodes) is also guided by the requirement of the experiment. The exposure time of the camera is usually defined by tuning the excitation power to maximize the brightness of the fluorescent object by maintaining an acceptable level of photo-bleaching throughout the experiment. Whenever single (or a limited number of) loci are tracked, it is desirable to reduce the size of the frame of the camera (or region of interest ROI), particularly for confocal laser scanning microscope confocal and sCMOS cameras (frame reduction is more complex for EM-CCD cameras, only achieved with an optical mask placed ahead of the sensor) to reduce the transfer time in between two consecutive images. Hence, for a given exposure time, the inter-frame interval can be optimized to reduce the consequences of photo-bleaching. The key parameter defining the quality of a recording is SNR of the target fluorescent region, as described in the following section.

\section{2: A few facts on the signal to noise ratio in single particle experiments}

The brightness of a fluorescent locus is estimated according to its SNR (see examples in the upper panel of Fig. 1A). This quantity is defined by the intensity of the locus corrected for the background signal $(A=4$ and 13 in the two upper graphs of Fig. $1 A)$ divided by the noise level in the background denoted $\Delta$ in Fig. 1A. This level is inferred from the standard deviation of the background signal. High SNR allows one to track an object with minimal localization error with a low false-detection rate, and thus to retrieve the maximal load of information out of a given experiment. In order to illustrate this statement, let us focus on the analysis of typical trajectories recorded for molecular transactions occurring in the nucleoplasm. We use the temporal evolution of the mean square displacement (MSD), which measures the average distance traveled by a target after a given time lag, as readout of these experiments (see section 2.3 for more information). In Figure 1B, we report diffusive MSD responses for GFP or RNA molecules in the nucleoplasm (green lines), as well as the typical sub-diffusive behavior detected for chromatin loci in yeast (blue line). Depending on the SNR of the object, tracking accuracy is limited spatially to a lower limit, which is presented by the five horizontal lines of the plot. It is not possible to detect spatial fluctuations smaller than these limits, due to positioning incertitude. For a locus characterized by an SNR of 3.4, chromosome spatial fluctuations or RNA diffusion cannot be sampled for time scales below $0.1 \mathrm{~s}$, but GFP diffusion is perfectly well characterized for this signal quality. In order to track chromatin down to $10 \mathrm{~ms}$, it is necessary to increase the SNR to $\sim 10$, by e.g. increasing the power of the light source, augmenting the gain in photodetectors, and adjusting the size of the frames. 


\section{3: Tracking and trajectory reconstruction}

DNA locus motion is analyzed using single particle tracking (SPT) techniques. SPT is based on the ability to accurately identify the center of a fluorescent spot at each time point of time-lapse imaging (2D or 3D) and connect those positions to reconstruct trajectories. Spatial resolution of SPT is not limited by the diffraction limit of $\sim 250 \mathrm{~nm}$ for GFP visualization, but by the signal-to-noise ratio (SNR) which depends on the number of photons detected and the noise generated by microscopic setup and detector. Automated detection based on Gaussian fitting is typically appropriate for high SNR, which have been rigorously compared in ref. (84), but other detection methods $(68,85)$ might be more robust and should be explored when SNR cannot be increased.

The standard approach to assess the level of localization precision consists in tracking simulated spots at a fixed position with a registered level of SNR. Simulated spots are generated using Matlab algorithms (or equivalent), in which we assign a Gaussian profile for the particle characteristics and add a Poisson noise to every pixel for background noise approximation. The size of the particle's Gaussian profile is dependent on the microscopy set-up as well as the camera's pixel size, especially for objects smaller than the pixel size. These parameters should be chosen to match experimental conditions. Note that the localization precision can also be measured experimentally by tracking fluorescent beads chemically bound to coverslips or with chemically-fixed FROS tagged cells, but artifacts associated to mechanical drift of the microscope during the acquisition should not be overlooked in this case. Simulated images are thus more relevant to assess the absolute positioning ("ground-truth" error), although subtle sources of errors in localization due to motion blur during camera acquisition (called dynamic errors; see ref. (86) for a review) are often neglected.

Regarding observation of chromosomal loci in living yeast, genes can be tracked in 2D with a resolution below $10 \mathrm{~nm}$, i.e. with an SNR larger than $\sim 30$, for time intervals $200 \mathrm{~ms}$ using FROS labeling (data not shown). It is often noted that chromosome loci move in the nuclear volume in 3D, and several techniques have been set up to track their motion along the three spatial directions, including, to cite but a few, micro-mirrors (87), double-helix point spread function microscope (88), astigmatic microscope (89), or confocal microscopy (82). So far, however, the advantages of 3D over 2D tracking remain unclear, because the acquisition of images in 3D reduces the temporal resolution and/or decreases the tracking accuracy, hence may limit the quality of the data retrieved from tracking experiments. Furthermore, the organization of chromosomes with respect to the focal plane of the objective is expected to be random (effects of gravity are a priori negligible), so the movement of chromosomes in the focal 
plane of the objective is similar to that in the third dimension. Therefore the information gathered from 3D tracking should essentially be identical to that collected in 2D, and the complications associated to 3D microscopy should not be rewarded with more accurate physical insights.

\section{4: Data analysis}

The diffusion coefficient $D$ and the velocity $v$ of the measured trajectories can be retrieved from the analysis of the mean square displacement (MSD):

$$
M S D_{1 D}(\tau)=\left\langle(x(t+\tau)-x(t))^{2}\right\rangle_{t}=2 D \tau+v^{2} \tau^{2}
$$

with $\tau$ the time interval, $x$ the position of the tracer, and $t$ the time variable for moving average.

The MSD corresponds to the average distance traveled after a given time lag $\tau$. Note that other statistical functions may be relevant for the analysis of random processes, including the velocity correlation function $(88,90)$ or the angle distribution between consecutive steps $(91)$. The motion of an isolated object diffusing in a viscous fluid is known to be associated to a linear temporal variation of the MSD with time, as reported in equation 1. Yet this model obviously constitutes an oversimplification, which can hardly be applied to probes moving in the nucleoplasm. In fact, the presence of obstacles with a structured or random spatial distribution (92) leads to deviation from the standard model with the MSD scaling as a power of time. This anomalous diffusive behavior, which has recently gained in popularity for chromosome fluctuation analysis in living cells (see table 2 and ref. (93)), is characterized with the following equation:

$$
M S D_{1 D}(\tau)=2 \Gamma \tau^{\alpha}
$$

with $\alpha$ the anomaly exponent and $\Gamma$ the amplitude of the MSD (units of $\mu \mathrm{m}^{2} / \mathrm{t}^{\alpha}$ ). Notably pioneering studies of chromosome dynamics have been initially analyzed with confined random walk models, i.e. normal diffusion in the small time limit and a plateau in the long time limit (see ref. (94) for a review). It is conceptually difficult to discriminate between confined random walk and anomalous diffusion based on fitting the MSD curve, because both models rely on two-fitting parameters. However it should be noted that models of polymer motion are nearly systematically associated to anomalous diffusion (95).

Importantly many mathematical and/or physical approaches have been developed to delineate between the most popular models of anomalous diffusion. Whereas normal diffusion originates from random collision with the solvent whatever the time scale, anomalous diffusion 
is associated to some degree of memory over time (94). For instance, the combination of random fluctuations with attachment of a polymer segment to its neighboring sites is associated to history effects in the trajectory of monomers because elastic forces hold back the monomer toward its previous position. More generally, models with short memory include continuoustime random walk and obstructed diffusion; on the other hand, fractional Brownian motion and fractional Levy stable motion correspond to a process with a long memory. Various tests have been established to differentiate between these classes of models, see e.g (96). Interestingly, these tests have been applied to study anomalous diffusion of telomeres (97) or yeast chromosomes (88), and they showed that chromosomes loci dynamics were consistent with fractional Brownian dynamics, which are very common for polymers. This result strongly supports the fact the analysis of the statistical properties of chromosome loci trajectories can be used to probe structural information of chromosomes.

The anomaly exponents describing the trajectories chromosome loci in yeast, bacteria, or mammalian cells (Table 2) have been consistently measured close to 0.5 . This behavior is very common for polymers for it corresponds to the Rouse regime. The Rouse model is expected to be relevant if the dynamics of a polymer is driven by elastic interactions along the chain with negligible long-range interactions mediated by solvent-flux (95). This approximation is consistent with the molecular environment of the nucleoplasm as the level of crowding $(98,99)$ should screen out long-range hydrodynamic flows. Think about spinning a fork in a spaghetti bowl: the fluid flow is dissipated at the vicinity of the cutlery. The Rouse model describes the chain as a series of beads connected by elastic springs, and it relies on two molecular parameters of the polymer, namely the monomer friction coefficient $\zeta$ and the length each segment which is defined by the Kuhn length $b$. Note that each Kuhn segment are associated with ball-joint connections, so that the orientation between consecutive monomers is random. Therefore the longer the Kuhn length, the stiffer the polymer. The expression for the MSD in 1D reads:

$$
M S D_{1 D}(\tau)=\left(\frac{4 b^{2} k_{B} T}{3 \pi \zeta}\right)^{0.5} \times \tau^{0.5}
$$

with $k_{B}$ and $T$ the Boltzmann constant, and the temperature, respectively. Note that the friction coefficient is related to the Kuhn length according to $\zeta=3 \pi \eta b$ with $\eta$ the viscosity for translational diffusion of monomers. The fact that the dynamics of monomers is determined by the polymer elastic properties implies that motion analysis offers a unique window to characterizing chromosome structural properties in vivo. The amplitude of the MSD has been estimated to $\Gamma \sim 0.01 \mu \mathrm{m}^{2} / \mathrm{s}^{0.5}$ for yeast chromosomes (100). Given that nucleoplasmic viscosity spans 4 to $7 \mathrm{mPa}$.s (101-103), we deduce that the Kuhn length is $\sim 1 \mathrm{~nm}$. This value of the Kuhn length expected to be on the order of 30 to $200 \mathrm{~nm}$ according to models and experiments (104) is much smaller than expected for a 10-nm fiber. This surprisingly high flexibility raises 
concerns about the consistency of this model and suggesting that additional physical effects, e.g. active forces out of equilibrium (90), may be considered. This step forward in the modeling remains a key issue for the structural description of chromosomes and for the understanding of how chromosome spatio-temporal dynamics are connected to their biological function in vivo.

\section{3: Mapping chromosome conformational dynamics by single/multi loci positioning}

Folding and physical data of chromatin conformation can be inferred from position of one or several specific DNA loci with respect to nuclear landmarks or relative to each other. Bulk labeling methods using fluorescent histones such as H2B-GFP or fluorescent dNTPs cannot inform on the path the underlying chromosome fiber follows. Increased labeling density and new, bright fluorescent dyes may ultimately, using super-resolution imaging techniques, yield a picture of this path (105) and systems biology approaches can inform on the probabilistic distribution of highly mobile DNA loci in vivo.

In yeast, two high throughput approaches based on automated, high precision tracking of single or multiple labelled sites have proven powerful in providing detailed information on the folding principles of chromosomes in the nucleus at steady state ((106-108), Fig. 2-3). They are described in the following paragraphs.

\section{1: Single locus localization in the nucleus in a large population of cells}

Investigations of gene localization are intrinsically limited by the diffraction blur of optical microscopy. Diffraction limits the spatial resolution to $200-500 \mathrm{~nm}$ at best, a major handicap considering the size of yeast nuclei of $2 \mu \mathrm{m}$ in diameter. For multiple point localization, this limitation can only be overcome with super-resolution detection, which so far remains a semiautomated and time-consuming technique, allowing the sampling of $\sim 100$ cells. A method coined 'gene-map' analysis (106) combines fully automated cell nuclei detection and single gene locus super-resolution 3D tracking (Fig. 2A). 3D coordinates of single gene loci tagged using tet0 FROS with respect to two nuclear landmarks, the nucleolus and the nuclear center, are automatically determined with a dedicated software. The positions can then be assembled in a single probabilistic representation of the gene's localization in nuclear space (Fig. 2B). The resolution of this gene localization is significantly better than the diffraction limit, and only 
limited by the SNR and the number of cells analyzed. Most genomic loci are confined to 'gene territories' which can be remodeled during transcriptional activation $(106,109)$. Overlay of different gene maps can distinguish preferential areas in which the respective DNA sites roam (Fig. 2C). These areas can be compared between different conditions or used to trace the path of a chromosome. For instance, by systematically inserting labels along chromosome XII, with 10 outside the rDNA and 3 in the rDNA region, the organization of chromosome XII, the largest yeast chromosome with $\sim 2 \mathrm{Mb}$ has been determined (107). The territory occupied by each tagged locus is relatively large compared to the overall volume of the nucleus and maps of two genes distant by $100 \mathrm{~kb}$ in genomic distance along the same chromosome arm readily overlap. Snapshots of a tagged locus in many different cells can inform on its 3D area of constraint, which also correlates with the average region explored by a locus through random fluctuations (107). The resulting data can be quantified with physics models, in which chromosomes are treated as polymer chains with volume exclusion and nuclear constraints defined by the nuclear envelope and the nucleolus $(110,111)$. This approach convincingly confirmed the Rabl-like organization of the yeast nucleus, an organization based on regrouping centromeres near the spindle pole body (SPB, a centrosome like structure embedded in the nuclear envelope) and clustering subsets of telomeres on the opposite end of the nucleus. Yet it did not allow determining chromatin structural parameters (see (38) for a review). The gene map method is readily applicable to fixed cells and DNA loci identified by FISH. Future improvements may permit analysis of relative positions and interdependence of several sites simultaneously, but it is so far difficult owing to the lack of high intensity fluorophores required for labeling multiple loci simultaneously.

\section{2: Multi-locus localization for chromosome conformation analysis}

In both yeast and mammalian cells, the regulation of position and associated dynamic behavior has been shown to be influenced by replication (81), transcription (44) as well as DNA repair (46). Two genomic sites can be labeled simultaneously to assess their relative position, in order to retrieve the propensity of two telomeres to coalesce (112), or to measure the relationship of their physical distance with respect to their separation on the linear chromosome, a measure useful to model the chromatin fiber (for a review see (113)). All these studies have so far been based on FROS labeling which allows to distinguish two sites by either different fluorescent intensities achieved using one short and one long array (128 vs. 256 repeats (73)), or two orthologous systems (lacO and tetO (114)). Note that a third site can be tagged using the bacterial lambda binding sequence $\lambda$ FROS $((115,116)$; Fig. 3A). Relative positions and angles can then be calculated by determining the position of three sites within the same nucleus. This 
approach has been instrumental in determining folding of chromosome III (320 kb) in yeast. Chromosome III folding appeared to be cell type specific, although preferential perinuclear localization was indistinguishable between the two cell types when analyzing individually tagged loci near the telomeres (HML and HMR) (47). Analysis of chromosome structure based on 3 point localization relies on dedicated software that automatically computes the 3D coordinates of each of the three loci from hundreds of nuclei (Fig. 3B). Distances and angles from the triangle can then be assembled in probabilistic representation of their relative position. Generated heatmaps inform on constraint between two loci relative to a third one (115). An inverse mathematical modeling approach has also been developed to generate survival zones for the three loci by fitting the experimental data iteratively. Survival zones define the area within which a locus can be positioned when physically bound to two other on the same chromosome fiber. In the case of chromosome III, it was possible to show that loci on the left arm of the chromosome had less freedom of position relative to the right arm of the chromosome in the alpha cell type than in the a cell type (Fig. 3C-D). Given the inability of the left arm of chromosome III to efficiently contact loci on the right one, chromosome conformation appears to relate to the mechanisms of homology search, the limiting step in homologous recombination. The geometrical ' 3 loci' algorithm is readily applicable to analyze position of three distinct sites at any distance in fixed and living cells.

\section{2: Real-time tracking of multiple loci}

Fitting the mean distance between two chromosomal sites with a polymer model based on a worm-like chain provides physical parameters of the chromatin fiber, one of which is the persistence length, or the Kuhn length (see above) divided by 2, a measure of polymer flexibility (114). This value suggested that, in situ, the fiber adopted a rather stiff conformation compatible with a $\sim 30 \mathrm{~nm}$ fiber. As the mere existence of such a fiber is more and more debated $(100,117)$, models have to be refined and additional parameters have to be computed. Chromatin is highly mobile and most loci explore a rather large subnuclear space. Hence, by using the mean distance motion and variability of measured distances are not taken into account. It is therefore highly desirable to combine multi-locus tracking to refine the analysis of distance averages. For instance, the distance between two telomeres of the same chromosome shows a general mean around $1 \mu \mathrm{m}$ (112), but telomeres of several short chromosomes move in a coordinated fashion ("dancing in tune") over a 5 min interval, and telomeres of distinct chromosomes do not (58). Correlated movement can be observed in $1 \mathrm{~s}$ intervals in 2D, but only in 3 min intervals in 3D. The duration of contact between two distinct loci can also be determined: for example the 
heterochromatic HML and HMR loci stay together on average $10 \mathrm{~s}$ (118). Simultaneous tracking of three or more discrete loci in close vicinity ( $<100 \mathrm{~kb}$ or less) has the greatest potential to describe fiber folding. An example in 2D is shown in Fig 3E-G, where the red labeled central locus, $20 \mathrm{~kb}$ and $50 \mathrm{~kb}$ separation to the green and blue one respectively on the same chromosome arm, moves with unconstrained amplitude, the three spots readily forming angles between 0 and 180 degrees. Hence, the chromatin fiber in a yeast interphase cell can bend around itself over distances of $<20 \mathrm{~kb}$ within less than $5 \mathrm{~min}$. Although such a study seems to be well-suited to derive insights on the flexibility of the fiber, it remains to be shown that static data (gene map or multi-locus data) can be bridged with fluctuation measurements. These questions clearly define an area of future research of genome biophysics which is essential to further elucidate the relationship between chromosome structural properties and their biological functions, as described in the following paragraph.

\section{4: Outlook - future developments}

The goal is now to elucidate how chromosome structural properties enable local or global reorganization of the nuclear architecture with respect to nuclear and cellular processes. This task amounts to define questions of systems biology where technology and physics can bring novel insights into nuclear organization. Its accomplishment will rely on technological developments in order to thoroughly sample the parameter "space" of molecular reactions involving chromosomes - as well as in physics -to build consistent and predictive chromosome models.

\subsection{Technological pipeline}

While the throughput of SPT has significantly improved over the past ten years, much effort remains to be carried out for screening multiple conditions and multiple colors. Let us first focus on multi-color fluorescence microscopy. Tracking two (or more) loci simultaneously in real time requires compromises between frequency, resolution, and duration (65): in 3D, the number of focal planes reduces the number of time frames; in 2D, one spot may move out of focus during the acquisition. The choice between 2D and 3D acquisition is hence subtle and the microscopy set-up to be employed is not straightforward. For instance, while confocal microscopes allow simultaneous use of several laser sources, spinning-disk or wide field 
microscope impose the choice between alternating wavelengths during stack acquisition or acquiring a stack for each wavelength. The first solution slows frequency depending on the speed change in light excitation and filter (wheel filters take $\sim 30 \mathrm{~ms}$ to switch), and the second reduces positional precision in particular for fast moving spots (if one stack takes $1 \mathrm{~s}$ to complete, the second stack will be acquired after $1 \mathrm{~s}$ ). Nevertheless confocal microscopy is not a fast acquisition technique, and scanning photo-multiplicators require reduction of the frame size, i.e. the number of pixels scanned (ROI) to gain enough speed for live cell tracking.

Automated Quantitative High-Throughput Microscopy (HTM) techniques are rapidly progressing. Confocal fluorescence micrographs of multiple fields can be captured from each microplate well of 96 or 384-well plates $(105,119)$, or from custom made microfluidic systems (120) generating massive amounts of data which will require custom MatLab algorithms and Image J plugins for automated, multiparametric analyses. Yet, while statistical analysis of single locus trajectories is now well-established with e.g. the MSD, the mathematical tools to use for multiple loci need to be developed. This task involves challenging computational and statistical approaches able to treat very noisy data from imaging very small nuclear segments.

\section{2: Chromosome structure and dynamics and their link to genome transactions}

In textbook models of DNA processing, chromosomes have been assumed to be immobile and proteins to scan the DNA backbone infinitely much faster (121-123). According to this target search mechanism, the folding of chromosomes plays an essential role to guide multi-target binding (124). The analysis of 3C data with polymer looping models seems to provide an excellent solution to better understand the orchestration of the expression of multiple genes simultaneously. Interestingly, the "immobility" hypothesis does not hold for chromatin repair pathways where distant chromosome sites have to interact. In this context the physics of chromatin motion and its interplay with chromatin structure remain to be clarified. Moreover it is widely believed that genes 'move' upon activating transcription, but whether the change in localization is a cause or a consequence of transcription and concomitant to mRNA production is not known. So far a few real time examples have shown a change in position as mRNA is produced $(41,42)$, but other studies of transcription activation have rather pointed to a reduced mobility (125) (Germier et al. unpublished). Also, it is well known that RNA polymerase forms clusters at active genes which are rather immobile (126), but quantitative studies of DNA motion as transcription proceeds need to be developed. Improving labeling and imaging techniques 
with shorter inserts, better SNR, greater resolution and speed will eventually allow tracking short-lived events, such as enhancer-promoter contacts. Together with consistent molecular modeling of chromatin, this convergence of physics and biology will clarify many aspects of the multiple regulation mechanisms of the genome. 


\section{Bibliography}

1. H. W. Mewes et al., Overview of the yeast genome. Nature. 387, 7-65 (1997).

2. S. Richards, It's more than stamp collecting: how genome sequencing can unify biological research. Trends in Genetics. 31, 411-421 (2015).

3. G. Vernikos, D. Medini, D. R. Riley, H. Tettelin, Ten years of pan-genome analyses. Current Opinion in Microbiology. 23, 148-154 (2015).

4. A. H. Paterson, Michael Freeling, H. Tang, X. Wang, Insights from the Comparison of Plant Genome Sequences. Annual Review of Plant Biology. 61, 349-372 (2010).

5. D. Baltimore, Our genome unveiled. Nature. 409, 814-816 (2001).

6. J. C. Venter, M. D. Adams, E. W. Myers, al et, The sequence of the human genome. Science. 291, 1304-1351 (2001).

7. I. Solovei et al., Spatial preservation of nuclear chromatin architecture during ThreeDimensional Fluorescence in Situ Hybridization (3D-FISH). Exp Cell Res. 276, 10-23 (2002).

8. F. L. Dily et al., Distinct structural transitions of chromatin topological domains correlate with coordinated hormone-induced gene regulation. Genes Dev. 28, 2151-2162 (2014).

9. A. Taddei, S. M. Gasser, Structure and Function in the Budding Yeast Nucleus. Genetics. 192, 107-129 (2012).

10. P. Fraser, W. Bickmore, Nuclear organization of the genome and the potential for gene regulation. Nature. 447, 413-417 (2007).

11. P. Therizols et al., Chromatin decondensation is sufficient to alter nuclear organization in embryonic stem cells. Science. 346, 1238-1242 (2014).

12. C. Lemaittre et al., Nuclear position dictates DNA repair pathway choice. Genes Dev. 28, 2450-2463 (2014).

13. P. Meister, A. Taddei, Building silent compartments at the nuclear periphery: a recurrent theme. Current Opinion in Genetics \& Development. 23, 96-103 (2013).

14. N. Gilbert et al., Chromatin Architecture of the Human Genome: Gene-Rich Domains Are Enriched in Open Chromatin Fibers. Cell. 118, 555-566 (2004).

15. E. Fussner, K. Ahmed, H. Dehghani, M. Strauss, D. P. Bazett-Jones, Cold Spring Harb Symp Quant Biol, in press, doi:10.1101/sqb.2010.75.012.

16. A. K. M. Shamsuddin, C.-K. Tang, Barr bodies in testis with Klinefelter syndrome. Urology. 15, 74-76 (1980).

17. C. Schöfer, K. Weipoltshammer, Gene dynamics and nuclear architecture during differentiation. Differentiation. 76, 41-56 (2008).

18. P. Fransz, J. H. de Jong, M. Lysak, M. R. Castiglione, I. Schubert, Interphase chromosomes in Arabidopsis are organized as well defined chromocenters from which euchromatin loops emanate. PNAS. 99, 14584-14589 (2002). 
19. K. A. Nasmyth, The regulation of yeast mating-type chromatin structure by SIR: An action at a distance affecting both transcription and transposition. Cell. 30, 567-578 (1982).

20. K. Weiss, R. T. Simpson, High-Resolution Structural Analysis of Chromatin at Specific Loci: Saccharomyces cerevisiae Silent Mating Type Locus HML $\alpha$. Mol. Cell. Biol. 18, 5392-5403 (1998).

21. A. Pombo, N. Dillon, Three-dimensional genome architecture: players and mechanisms. Nat Rev Mol Cell Biol. 16, 245-257 (2015).

22. Y. Lahbib-Mansais et al., Expressed alleles of imprinted IGF2, DLK1 and MEG3 colocalize in 3D-preserved nuclei of porcine fetal cells. BMC Cell Biology. 17, 35 (2016).

23. H. Chen et al., Functional organization of the human 4D Nucleome. PNAS. 112, 8002-8007 (2015).

24. T. Cremer et al., The 4D nucleome: Evidence for a dynamic nuclear landscape based on coaligned active and inactive nuclear compartments. FEBS Letters. 589, 2931-2943 (2015).

25. T. Sexton, G. Cavalli, The Role of Chromosome Domains in Shaping the Functional Genome. Cell. 160, 1049-1059 (2015).

26. S. Tashiro, C. Lanctôt, The International Nucleome Consortium. Nucleus. 6, 89-92 (2015).

27. S. M. Gasser, Nuclear Architecture: Past and Future Tense. Trends in Cell Biology. 26, 473475 (2016).

28. A. Denker, W. de Laat, A Long-Distance Chromatin Affair. Cell. 162, 942-943 (2015).

29. F. Ay, W. S. Noble, Analysis methods for studying the 3D architecture of the genome. Genome Biology. 16, 183 (2015).

30. A. S. Belmont, Large-scale chromatin organization: the good, the surprising, and the still perplexing. Current Opinion in Cell Biology. 26, 69-78 (2014).

31. A. Cournac, H. Marie-Nelly, M. Marbouty, R. Koszul, J. Mozziconacci, Normalization of a chromosomal contact map. BMC Genomics. 13, 436 (2012).

32. E. Yaffe, A. Tanay, Probabilistic modeling of Hi-C contact maps eliminates systematic biases to characterize global chromosomal architecture. Nat Genet. 43, 1059-1065 (2011).

33. S. Shachar, T. C. Voss, G. Pegoraro, N. Sciascia, T. Misteli, Identification of Gene Positioning Factors Using High-Throughput Imaging Mapping. Cell. 162, 911-923 (2015).

34. I. Williamson et al., Spatial genome organization: contrasting views from chromosome conformation capture and fluorescence in situ hybridization. Genes Dev. 28, 2778-2791 (2014).

35. L. Giorgetti, E. Heard, Closing the loop: 3C versus DNA FISH. Genome Biol. 17, 215 (2016).

36. J. Mateos-Langerak et al., Spatially confined folding of chromatin in the interphase nucleus. Proc Natl Acad Sci U S A. 106, 3812-3817 (2009). 
37. H. Yokota, G. van den Engh, J. E. Hearst, R. K. Sachs, B. J. Trask, Evidence for the Organization of Chromatin in Megabase Pair-sized Loops Arranged along a Random Walk Path in the Humand G0/G1 Interphase Nucleus. J Cell Biol. 130, 1239-1249 (1995).

38. S. Huet et al., Relevance and limitations of crowding, fractal, and polymer models to describe nuclear architecture. Int Rev Cell Mol Biol. 307, 443-479 (2014).

39. W. . Marshall et al., Interphase chromosomes undergo constrained diffusional motion in living cells. Current Biology. 7, 930-939 (1997).

40. V. Levi, Q. Ruan, M. Plutz, A. S. Belmont, E. Gratton, Chromatin Dynamics in Interphase Cells Revealed by Tracking in a Two-Photon Excitation Microscope. Biophysical Journal. 89, 4275-4285 (2005).

41. C.-H. Chuang et al., Long-Range Directional Movement of an Interphase Chromosome Site. Current Biol. 16, 825-831 (2006).

42. T. Tumbar, A. S. Belmont, Interphase movements of a DNA chromosome region modulated by VP16 transcriptional activator. Nat Cell Biol. 3, 134-139 (2001).

43. A. Taddei et al., Nuclear pore association confers optimal expression levels for an inducible yeast gene. Nature. 441, 774-778 (2006).

44. G. G. Cabal et al., SAGA interacting factors confine sub-diffusion of transcribed genes to the nuclear envelope. Nature. 441, 770-773 (2006).

45. J. R. Chubb, S. Boyle, P. Perry, W. A. Bickmore, Chromatin Motion Is Constrained by Association with Nuclear Compartments in Human Cells. Current Biology. 12, 439-445 (2002).

46. M. Lisby, U. H. Mortensen, R. Rothstein, Colocalization of multiple DNA double-strand breaks at a single Rad52 repair center. Nat Cell Biol. 5, 572-577 (2003).

47. K. Bystricky et al., Regulation of nuclear positioning and dynamics of the silent mating type loci by the yeast Ku70/Ku80 complex. Mol Cell Biol. 29, 835 (2009).

48. S. Nagai et al., Functional Targeting of DNA Damage to a Nuclear Pore-Associated SUMODependent Ubiquitin Ligase. Science. 322, 597-602 (2008).

49. C. Lemaître, E. Soutoglou, Double strand break (DSB) repair in heterochromatin and heterochromatin proteins in DSB repair. DNA Repair. 19, 163-168 (2014).

50. Y. Avivi et al., Reorganization of specific chromosomal domains and activation of silent genes in plant cells acquiring pluripotentiality. Developmental Dynamics. 230, 12-22 (2004).

51. S. Chambeyron, W. A. Bickmore, Chromatin decondensation and nuclear reorganization of the HoxB locus upon induction of transcription. Genes \& Dev. 18, 1119-1130 (2004).

52. A. Zidovska, D. A. Weitz, T. J. Mitchison, Micron-scale coherence in interphase chromatin dynamics. PNAS. 110, 15555-15560 (2013).

53. T. Misteli, Protein dynamics: implications for nuclear architecture and gene expression. Science. 291, 843-847 (2001). 
54. R. Bruinsma, A. Y. Grosberg, Y. Rabin, A. Zidovska, Chromatin Hydrodynamics. Biophysical Journal. 106, 1871-1881 (2014).

55. D. Gerlich, J. Ellenberg, 4D imaging to assay complex dynamics in live specimens. Nat Cell Biol. Suppl:, S14-S9 (2003).

56. J. Walter, L. Schermelleh, M. Cremer, S. Tashiro, T. Cremer, Chromosome order in HeLa cells changes during mitosis and early G1, but is stably maintained during subsequent interphase stages. J Cell Biol. 160, 685-697 (2003).

57. J. Liu, P.-A. Vidi, S. A. Lelièvre, J. M. K. Irudayaraj, Nanoscale histone localization in live cells reveals reduced chromatin mobility in response to DNA damage. J Cell Sci. 128, 599604 (2015).

58. K. Bystricky, T. Laroche, G. Van Houwe, M. Blaszczyk, S. M. Gasser, Chromosome looping in yeast. JCB. 168, 375 (2005).

59. E. Kepten, A. Weron, I. Bronstein, K. Burnecki, Y. Garini, Uniform Contraction-Expansion Description of Relative Centromere and Telomere Motion. Biophysical Journal. 109, 14541462 (2015).

60. J. R. Dixon et al., Topological domains in mammalian genomes identified by analysis of chromatin interactions. Nature. 485, 376-380 (2012).

61. S. Wang et al., Spatial organization of chromatin domains and compartments in single chromosomes. Science, aaf8084 (2016).

62. D. Zink, N. Sadoni, E. Stelzer, Visualizing chromatin and chromosomes in living cells. Methods. 29, 42-50 (2003).

63. S. Hihara et al., Local Nucleosome Dynamics Facilitate Chromatin Accessibility in Living Mammalian Cells. Cell Reports. 2, 1645-1656 (2012).

64. J. Lacroix et al., Small, in press, doi:10.1002/smll.201503795.

65. K. Bystricky, Chromosome dynamics and folding in eukaryotes: Insights from live cell microscopy. FEBS Letters. 589, 3014-3022 (2015).

66. A. F. Straight, A. S. Belmont, C. C. Robinett, A. W. Murray, GFP tagging of budding yeast chromosomes reveals that protein-protein interactions can mediate sister chromatid cohesion. Current Biology. 6, 1599-1608 (1996).

67. C. C. Robinett et al., In vivo localization of DNA sequences and visualization of large-scale chromatin organization using lac operator/repressor recognition. The Journal of Cell Biology. 135, 1685 (1996).

68. I. Lassadi, K. Bystricky, in DNA Recombination, H. Tsubouchi, Ed. (Humana Press, 2011; http://dx.doi.org/10.1007/978-1-61779-129-1_29), Methods in Molecular Biology, pp. 499-522.

69. I. Loïodice, M. Dubarry, A. Taddei, in Current Protocols in Cell Biology (John Wiley \& Sons, Inc., 2014; http://onlinelibrary.wiley.com/doi/10.1002/0471143030.cb2217s62/abstract). 
70. M. Dubarry, I. Loïodice, C. L. Chen, C. Thermes, A. Taddei, Tight protein-DNA interactions favor gene silencing. Genes Dev. 25, 1365-1370 (2011).

71. M. E. Tanenbaum, L. A. Gilbert, L. S. Qi, J. S. Weissman, R. D. Vale, A Protein-Tagging System for Signal Amplification in Gene Expression and Fluorescence Imaging. Cell. 159, 635-646 (2014).

72. H. Saad et al., DNA Dynamics during Early Double-Strand Break Processing Revealed by Non-Intrusive Imaging of Living Cells. PLoS Genetics. 10 (2014).

73. P. Simon, P. Houston, J. Broach, Directional bias during mating type switching in Saccharomyces is independent of chromosomal architecture. The EMBO Journal. 21, 2282-2291 (2002).

74. D. A. Bressan, J. Vazquez, J. E. Haber, Mating type-dependent constraints on the mobility of the left arm of yeast chromosome III. J Cell Biol. 164, 361-371 (2004).

75. P. M. Carlton et al., Fast live simultaneous multiwavelength four-dimensional optical microscopy. Proc Nat Acad Sci USA. 107, 16016 (2010).

76. B. Chen et al., Dynamic imaging of genomic loci in living human cells by an optimized CRISPR/Cas system. Cell. 155, 1479-91 (2013).

77. H. Ma et al., Multicolor CRISPR labeling of chromosomal loci in human cells. Proceedings of the National Academy of Sciences, 201420024 (2015).

78. H. Ma, P. Reyes-Gutierrez, T. Pederson, Visualization of repetitive DNA sequences in human chromosomes with transcription activator-like effectors. PNAS. 110, 2104821053 (2013).

79. Y. Miyanari, C. Ziegler-Birling, M.-E. Torres-Padilla, Live visualization of chromatin dynamics with fluorescent TALEs. Nat Struct Mol Biol. 20, 1321-1324 (2013).

80. S. Shao et al., Long-term dual-color tracking of genomic loci by modified sgRNAs of the CRISPR/Cas9 system. Nucl. Acids Res., gkw066 (2016).

81. P. Heun, T. Laroche, K. Shimada, P. Furrer, S. M. Gasser, Chromosome dynamics in the yeast interphase nucleus. Science. 294, 2181 (2001).

82. V. Levi, E. Gratton, in Single Particle Tracking and Single Molecule Energy Transfer, C. Bräuchle, D. C. Lamb, J. Michaelis, Eds. (Wiley-VCH Verlag GmbH \& Co. KGaA, 2009; http://onlinelibrary.wiley.com/doi/10.1002/9783527628360.ch1/summary), pp. 1-24.

83. J. Vermot, S. E. Fraser, M. Liebling, Fast fluorescence microscopy for imaging the dynamics of embryonic development. HFSP Journal. 2, 143-155 (2008).

84. N. Chenouard et al., Objective comparison of particle tracking methods. Nat Meth. 11, 281-289 (2014).

85. I. F. Sbalzarini, P. Koumoutsakos, Feature point tracking and trajectory analysis for video imaging in cell biology. J Struct Biol. 151, 182-195 (2005).

86. T. Savin, P. S. Doyle, Static and Dynamic Errors in Particle Tracking Microrheology. Biophysical Journal. 88, 623-638 (2005). 
87. H. Hajjoul, S. Kocanova, I. Lassadi, K. Bystricky, A. Bancaud, Lab-on-Chip for fast 3D particle tracking in living cells. Lab on a Chip. 9, 3054-3058 (2009).

88. M. P. Backlund, R. Joyner, K. Weis, W. E. Moerner, Correlations of three-dimensional motion of chromosomal loci in yeast revealed by the double-helix point spread function microscope. Mol. Biol. Cell. 25, 3619-3629 (2014).

89. V. Récamier et al., Single cell correlation fractal dimension of chromatin. Nucleus. 5, 75-84 (2014).

90. S. C. Weber, A. J. Spakowitz, J. A. Theriot, Nonthermal ATP-dependent fluctuations contribute to the in vivo motion of chromosomal loci. Proc Nat Acad Sci USA. 109, 73387343 (2012).

91. I. Izeddin et al., Single-molecule tracking in live cells reveals distinct target-search strategies of transcription factors in the nucleus. eLife. 3, e02230 (2014).

92. M. J. Saxton, Single Particle Tracking. Fundamental Concepts in Biophysics, 1-33 (2009).

93. C. Manzo, M. F. Garcia-Parajo, A review of progress in single particle tracking: from methods to biophysical insights. Rep. Prog. Phys. 78, 124601 (2015).

94. R. Metzler, J. Klafter, The random walk's guide to anomalous diffusion: a fractional dynamics approach. Physics Reports. 339, 1-77 (2000).

95. M. Doi, S. F. Edwards, The theory of polymer dynamics (Oxford University Press, USA, 1988).

96. K. Burnecki, M. Muszkieta, G. Sikora, A. Weron, Statistical modelling of subdiffusive dynamics in the cytoplasm of living cells: A FARIMA approach. EPL (Europhysics Letters). 98, 10004 (2012).

97. K. Burnecki et al., Universal Algorithm for Identification of Fractional Brownian Motion. A Case of Telomere Subdiffusion. Biophysical Journal. 103, 1839-1847 (2012).

98. C. Chen et al., Budding yeast chromatin is dispersed in a crowded nucleoplasm in vivo. bioRxiv, 056465 (2016).

99. R. Hancock, Structures and functions in the crowded nucleus: new biophysical insights. Front. Phys. 2, 53 (2014).

100. H. Hajjoul et al., High throughput chromatin motion tracking in living yeast reveals the flexibility of the fiber throughout the genome. Genome research. 23, 1829-1838 (2013).

101. A. S. Verkman, Solute and macromolecule diffusion in cellular aqueous compartments. Trends in Biochemical Sciences. 27, 27-33 (2002).

102. O. Seksek, J. Biwersi, A. S. Verkman, Translational diffusion of macromolecule-sized solutes in cytoplasm and nucleus. J Cell Biol. 138, 131-142 (1997).

103. J. Beaudouin, F. Mora-Bermudez, T. Klee, N. Daigle, J. Ellenberg, Dissecting the contribution of diffusion and interactions to the mobility of nuclear proteins. Biophys J. 90, 1878-94 (2006).

104. H. Schiessel, The Physics of Chromatin. J Phys : Condens Matter. 15, R699-R774 (2003). 
105. B. J. Beliveau et al., Single-molecule super-resolution imaging of chromosomes and in situ haplotype visualization using Oligopaint FISH probes. Nat Commun. 6, 7147 (2015).

106. A. B. Berger et al., High-resolution statistical mapping reveals gene territories in live yeast. Nat Meth. 5, 1031-1037 (2008).

107. B. Albert et al., Systematic characterization of the conformation and dynamics of budding yeast chromosome XII. Journal of Cell Biology. 202, 201-210 (2013).

108. I. Lassadi, A. Kamgoué, I. Goiffon, N. Tanguy-le-Gac, K. Bystricky, Differential Chromosome Conformations as Hallmarks of Cellular Identity Revealed by Mathematical Polymer Modeling. PLOS Comput Biol. 11, e1004306 (2015).

109. P. Therizols, T. Duong, B. Dujon, C. Zimmer, E. Fabre, Chromosome arm length and nuclear constraints determine the dynamic relationship of yeast subtelomeres. Proceedings of the National Academy of Sciences. 107, 2025 (2010).

110. H. Wong et al., A predictive computational model of the dynamic 3D interphase nucleus. Curr Biol. 22, 1881-1890 (2012).

111. H. Tjong, K. Gong, L. Chen, F. Alber, Physical tethering and volume exclusion determine higher-order genome organization in budding yeast. Genome Res. 22, 1295-1305 (2012).

112. H. Schober et al., Controlled exchange of chromosomal arms reveals principles driving telomere interactions in yeast. Genome research. 18, 261 (2008).

113. L. Mirny, The fractal globule as a model of chromatin architecture in the cell. Chromosome Research. 19, 37-51 (2011).

114. K. Bystricky, P. Heun, L. Gehlen, J. Langowski, S. M. Gasser, Long range compaction and flexibility of interphase chromatin in budding yeast analysed by high-resolution imaging techniques. Proc Natl Acad Sci U S A. 101, 16495-16500 (2004).

115. I. Lassadi, A. Kamgoué, I. Goiffon, N. Tanguy-le-Gac, K. Bystricky, Differential chromosome conformations as hallmarks of cellular identity revealed by mathematical polymer modeling. PLoS Computational Biology (2015), doi:10.1371/journal.pcbi.1004306.

116. R. A. Fekete, D. K. Chattoraj, A cis-acting sequence involved in chromosome segregation in Escherichia coli. Molecular Microbiology. 55, 175-183 (2005).

117. K. Maeshima, S. Hihara, M. Eltsov, Chromatin structure: does the 30-nm fibre exist in vivo? Current Opinion in Cell Biology (2010).

118. A. Miele, K. Bystricky, J. Dekker, Yeast silent mating type loci form heterochromatic clusters through silencer protein-dependent long-range interactions. PLoS Genetics. 5 (2009).

119. V. Roukos et al., Spatial dynamics of chromosme translocations in living cells. Science. 341, 660-664 (2013).

120. K. R. King et al., A high-throughput microfluidic real-time gene expression living cell array. Lab Chip. 7, 77-85 (2006).

121. D. Normanno et al., Probing the target search of DNA-binding proteins in mammalian cells using TetR as model searcher. Nat Commun. 6, 7357 (2015). 
122. P. H. von Hippel, O. G. Berg, Facilitated target location in biological systems. J Biol Chem. 264, 675-678 (1989).

123. J. Elf, G.-W. Li, X. S. Xie, Probing transcription factor dynamics at the single molecule level in a living cell. Science. 316, 1191-1194 (2007).

124. J. Dekker, L. Mirny, The 3D Genome as Moderator of Chromosomal Communication. Cell. 164, 1110-1121 (2016).

125. H. Ochiai, T. Sugawara, T. Yamamoto, Simultaneous live imaging of the transcription and nuclear position of specific genes. Nucl. Acids Res. 43, e127-e127 (2015).

126. X. Darzacq et al., Imaging transcription in living cells. Annual review of biophysics. $\mathbf{3 8}$, 173-196 (2009).

127. C. Molenaar, A. Abdulle, A. Gena, H. J. Tanke, R. W. Dirks, Poly(A)+ RNAs roam the cell nucleus and pass through speckle domains in transcriptionally active and inactive cells. $J$ Cell Biol. 165, 191-202 (2004).

128. T. Lebeaupin, H. Sellou, G. Timinszky, S. Huet, Chromatin dynamics at DNA breaks: what, how and why? AIMS Biophysics. 2, 458-475 (2015).

129. D. Gerlich et al., Global chromosome positions are transmitted through mitosis in mammalian cells. Cell. 112, 751-764 (2004).

130. I. Bronstein et al., Transient anomalous diffusion of telomeres in the nucleus of mammalian cells. Phys Rev Lett. 103, 18102 (2009).

131. N. Dimitrova, Y.-C. M. Chen, D. L. Spector, T. de Lange, 53BP1 promotes non-homologous end joining of telomeres by increasing chromatin mobility. Nature. 456, 524-528 (2008).

132. J. J. Wanat et al., Csm4, in Collaboration with Ndj1, Mediates Telomere-Led Chromosome Dynamics and Recombination during Yeast Meiosis. PLOS Genet. 4, e1000188 (2008).

133. E. Bertrand, P. S. Chartrand, S. M. Shenoy, R. H. Singer, R. M. Long, Localization of ASH1 mRNA particles in living yeast. Mol Cell. 2, 437-445 (1998).

134. S. Hocine, P. Raymond, D. Zenklusen, J. A. Chao, R. H. Singer, Single-molecule analysis of gene expression using two-color RNA labeling in live yeast. Nat Meth. 10, 119-121 (2013).

135. T. J. Stasevich et al., Regulation of RNA polymerase II activation by histone acetylation in single living cells. Nature. 516, 272-275 (2014).

136. S. C. Weber, A. J. Spakowitz, J. A. Theriot, Bacterial Chromosomal Loci Move Subdiffusively through a Viscoelastic Cytoplasm. Phys Rev Lett. 104, 238102 (2010).

137. A. Javer et al., Short-time movement of E. coli chromosomal loci depends on coordinate and subcellular localization. Nat Commun. 4, 3003 (2013).

138. R. Wang, J. Mozziconacci, A. Bancaud, O. Gadal, Principles of chromatin organization in yeast: relevance of polymer models to describe nuclear organization and dynamics. Current Opinion in Cell Biology. 34, 54-60 (2015). 
139. I. Bronshtein et al., Loss of lamin A function increases chromatin dynamics in the nuclear interior. Nature Communications. 6, 8044 (2015). 


\section{Figure Legends}

Figure 1: Particle tracking and positioning error a) The upper panel shows four fluorescence micrographs of $0.4 \mu \mathrm{m}$ nanoparticles imaged with a wide field microscope equipped with a 100X objective (NA=1.4). The different SNR are obtained by exposing the nanoparticles to variable doses of illumination. The two associated graphs correspond to the intensity vs. pixel position along the respective green and red arrows drawn on the two micrographs. Note that the color of the datasets is associated to the arrows and the black lines are Gaussian fits of width 3.7 pixels. b) The graph represents the temporal variation of the MSD for three typical situations in the nucleoplasm. In green, we consider free diffusion of GFP (103) or RNA (127), and in blue the sub-diffusive response detected for chromosome loci (100). The five horizontal lines correspond to the lower limits of detection for variable values of the SNR, as indicated in inset. Note that these horizontal lines are deduced from simulations of fixed particles, which produce noisy black traces, as represented for SNR=33.7.

Figure 2 : A systems biology approach to study gene position. a) Yeast nuclei were imaged in 3D acquiring $21 \mathrm{z}$ planes at $250 \mathrm{~nm}$ intervals using a spinning disk microscope. The nuclear envelope (green) and the nucleolus (red) were labeled by expressing endogenously tagged fusion proteins GFP-Nup49 (nucleoporin; green) and Nop1-mcherry, respectively. The DNA locus of interest is labeled by inserting an array of 256 TetO and expressing TetR-GFP. Excitation time was 200 ms. An example of a field of $\sim 100$ nuclei is shown. b) An example of a gene map showing the probabilistic distribution of the imaged DNA locus from thousands of nuclei. Only round shaped nuclei are analyzed. c) Gene maps obtained from different yeast strains in which distinct DNA loci were labeled (here loci along yeast chromosome XII at positions 14, 70 and 120 $\mathrm{kb}$ from the left telomere and the centromere (cen)) can be overlaid. d) The relative position of the gene maps allows reconstructing the path of chromosomes with respect to nuclear landmarks.

Figure 3 : Multi-loci labeling to determine chromosome conformation and folding. a) Live yeasts were imaged in 3D acquiring $21 \mathrm{z}$ stacks separated by $250 \mathrm{~nm}$ intervals using a wide-field microscope. Three distinct loci were labeled simultaneously by inserting arrays of LacO, TetO and $\lambda O$ (next to the three mating type loci, HML, MAT and HMR, on chromosome III), and visualized by co-expressing CFP-LacI, TetR-mcherry and $\lambda I$-YFP. Excitation times were 300-400 ms. b) The center of gravity of each spot and the distance between the three spots was 
determined automatically from hundreds of nuclei. c) An inverse mathematical modelling approach was used to determine the areas in which each DNA locus can roam when linked to the 2 others (Survival zones in red, blue and green contours). d) Relative positions of the survival zones can be compared between two cell types. e) A triple labeled single yeast cell was imaged in 2D in real time, 1 image/ 10 seconds over 5 minutes. Here, FROS arrays were inserted along the same chromosome arm 17, 74 and $90 \mathrm{~kb}$ from the left telomere of chromosome III. f) The angle formed at the central red locus formed between the blue and green one is shown from 350 cells. All angles, as represented in panel $\mathbf{g}$ ) and $\mathbf{h}$ ), are possible, suggesting that the red locus can move with respect to the two others without constraint. 


\section{Glossary:}

- 3C: chromosome conformation capture

- TADs : topologically associated domains; TADs represent a chromatin domain within which physical interactions occur more frequently than bulk of the genome

- SNR signal to noise ratio;

- FROS :fluorescent repressor operator system; FROS combines the expression of a bacterial repressor fused to a fluorescent protein and the integration of operator sequence as tandem arrays at a specific locus

- TALE: transcription activator like effector proteins

- ANCHOR: ANCHOR system is a bipartite system composed of ANCH sequences (derived from bacterial ParS site) that contains nucleation seeds for the binding of OR proteins (derived from ParB protein). Based on Bacteria chromosome partition systems (Burkholderiaecae), three or more ANCHOR systems can be used

- SunTAg: SUperNova tagging system; Sun-Tag is based on antibody to a multimerized protein scaffold

- OMX microscope: "Optical Microscope eXperimental" is a platform for multimodal, multichannel wide-field imaging. OMX can be used for three-dimensional structured illumination microscopy (3D-SIM)

- FISH: Fluorescent in situ hybridization

- sCMOS or CCD/CMOS cameras: Detectors consists of complementary metal-oxidesemiconductor (CMOS) readout integrated circuits that are bump bonded to a CCD imaging substrat; sCMOS camera have small pixel size and high sensitivity

- EM-CCD: electron-multiplying -charge-coupled device. EM-CCD camera have high sensitivity, low noise, but larger pixel size and are slower than sCMOS detector.

- MSD: mean square displacement; For every time interval (s), the square of the mean displacement observed $(\mu \mathrm{m} 2)$ is calculated. Confinement of a locus in areas of the nucleus appears as a plateau at longer time scale, defining a radius of constrains (expressed in $\mu \mathrm{m}$, see gene territories)

- $\quad$ SPT : single particle tracking

- fBM: Fractional Brownian motion

- OD : Obstructed diffusion

- FLSM: fractional Levy stable motion

- Gene territories: Volume $\left(\mu \mathrm{m}^{3}\right)$ in which $50 \%$ of gene's loci are confined in a cell population analysis 
- rDNA: ribosomal DNA; genes coding ribosomal DNA are organized in loci in direct tandem head-to-tail repeat (100-200 fold).

- HML and HMR: Hidden MAT Left and right; Correspond to Silent Mating type cassette left or right.

- ROI : Region of interest

- HTM: High-throughput microscopy 
Tables:

Table1: Methods for tracking chromatin motion in living cells

\begin{tabular}{|c|c|c|c|}
\hline Method & $\begin{array}{l}\text { Origin [ } n=\text { number of } \\
\text { systems in use] }\end{array}$ & Sequences labelled & $\begin{array}{l}\text { Reference } *[\text { first } \\
\text { application to motion } \\
\text { analysis, selected examples] }\end{array}$ \\
\hline $\begin{array}{l}\text { Fluorescent } \\
\text { dNTPs }\end{array}$ & dNTP $(n=4)$, BrdU & $\begin{array}{l}\text { Bulk labelling; whole genome } \\
\text { single cell injections or 'rubbing'; } \\
\text { transient labeling }\end{array}$ & $(128)$ \\
\hline Histones & H2B-GFP etc. $(n>4)$ & $\begin{array}{l}\text { Bulk labelling; whole genome } \\
\text { Photoactivatable fusion allows } \\
\text { activating individual chromosomes, } \\
\text { chromosome territories }\end{array}$ & $(52,63,89,129)$ \\
\hline $\begin{array}{l}\text { Chromatin } \\
\text { associated } \\
\text { proteins }\end{array}$ & $\begin{array}{l}\text { Rap1-GFP (yeast), TRF1- } \\
\text { GFP (mammalian), Rad52- } \\
\text { GFP, mch-53BP1 others }\end{array}$ & $\begin{array}{l}\text { Visualization of repetitive sequences } \\
\text { (telomeres, centromeres) or protein } \\
\text { accumulation at double strand breaks }\end{array}$ & $(59,130-132)$ \\
\hline $\mathrm{MS} 2 / \mathrm{MCP}$ & $\begin{array}{l}\text { mRNA } \\
n=2(M S 2, P P 7)\end{array}$ & $\begin{array}{l}\text { Visualization of specific nascent } \\
\text { mRNA transcripts bearing repetitive } \\
\text { stem loop sequences (genome } \\
\text { editing); indirect, close to gene }\end{array}$ & $(126,133,134)$ \\
\hline $\begin{array}{l}\text { Fluorescent } \\
\text { Antibody } \\
\text { fragments } \\
\text { (Fab) }\end{array}$ & $\begin{array}{l}\text { Antibody (unlimited but } \\
\text { synthesis laborious) }\end{array}$ & $\begin{array}{l}\text { Labelling of histones or proteins } \\
\text { associated with repetitive gene } \\
\text { arrays; positional information }\end{array}$ & $(135)$ \\
\hline $\begin{array}{l}\text { FROS } \\
\text { (Fluorescent } \\
\text { repressor } \\
\text { operator } \\
\text { system) }\end{array}$ & $\begin{array}{l}\text { n=3; Lac0/lacI (E.coli } \\
\text { chromosome); TetO/TetR } \\
\text { (Tn10 transposon } \\
\text { tetracycline gene); LO/LCi } \\
\text { (phage lambda); } \\
\text { potentially other bacteria } \\
\text { operons (ie. lexA; } \\
\text { cu0/CymR; ycnKJI) }\end{array}$ & $\begin{array}{l}\text { Visualization of specific DNA sites by } \\
\text { inserting arrays of operator sites } \\
\text { (genome editing) }\end{array}$ & $(39,66,115)$ \\
\hline $\begin{array}{l}\text { TALE } \\
\text { (transcription } \\
\text { activator like } \\
\text { effector } \\
\text { proteins) }\end{array}$ & $\begin{array}{l}\mathrm{N}=2 \text { but potentially } \infty \\
\text { Xanthomonas oryzae } \\
\text { transcription activator }\end{array}$ & $\begin{array}{l}\text { Specific natural repetitive sequences } \\
\text { (ie. telomeres, centromeres) }\end{array}$ & $(78,79)$ \\
\hline $\begin{array}{l}\text { CRISPR/ } \\
\text { dCas9 }\end{array}$ & $\begin{array}{l}\mathrm{N}=3 \text {; Streptococcus } \\
\text { pyrogenes (Sp), Neisseria } \\
\text { meningitides (Nm), and } \\
\text { Streptococcus } \\
\text { thermophilus (St1) }\end{array}$ & $\begin{array}{l}\text { Repetitive sequences or numerous } \\
\text { sites recognized by small guide RNAs } \\
(\mathrm{n}>\sim 70) \text {; transient labeling; }\end{array}$ & $(76,77)$ \\
\hline SunTag & $\begin{array}{l}\text { Antibody to a multimerized } \\
\text { protein scaffold }\end{array}$ & $\begin{array}{l}\text { Visualization of specific DNA sites by } \\
\text { inserting a protein binding site } \\
\text { (genome editing) }\end{array}$ & (71) \\
\hline $\begin{array}{l}\text { ANCHOR } \\
\text { (ParB/INT) }\end{array}$ & $\begin{array}{l}\mathrm{N}=3 \text { (potentially more) } \\
\text { Bacteria chromosome } \\
\text { partition systems } \\
\text { (Burkholderiaecae) }\end{array}$ & $\begin{array}{l}\text { Visualization of specific DNA sites by } \\
\text { inserting a short non-repetitive } \\
\text { sequence (genome editing) }\end{array}$ & (72), Germier et al. in preparation \\
\hline
\end{tabular}


Table 2. Overview on recent studies on chromosome dynamics.

\begin{tabular}{|c|c|c|c|c|}
\hline & Study & $\begin{array}{l}\text { Anomaly } \\
\text { exponent }\end{array}$ & Time scale & Interpretation \\
\hline \multirow{3}{*}{ 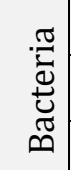 } & $(136)$ & 0.4 & $1-200 \mathrm{~s}$ & Rouse in visco-elastic media \\
\hline & $(90)$ & 0.4 & $1-200 \mathrm{~s}$ & "Superthermal" response \\
\hline & $(137)$ & 0.4 & $0.1-10 \mathrm{~s}$ & Fractional Langevin motion \\
\hline \multirow{7}{*}{ 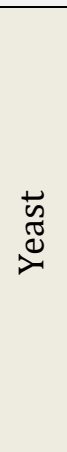 } & (39) & $\begin{array}{c}1 \text { and } \\
\text { plateau }\end{array}$ & $12-500 \mathrm{~s}$ & $\begin{array}{c}\text { Confined random motion } \\
\text { Reptation for diffusion coefficient }\end{array}$ \\
\hline & $(44)$ & $0.4-0.5$ & $4-100 s$ & Subdiffusion \\
\hline & $(87)$ & 0.4 & $0.2-4 \mathrm{~s}$ & Sub diffusion \\
\hline & $(107)$ & $0.5 \pm 0.07$ & $0.1-200 \mathrm{~s}$ & Rouse model \\
\hline & $(100)$ & $0.52 \pm 0.07$ & $0.02-500 \mathrm{~s}$ & Rouse model \\
\hline & $(88)$ & 0.7 & $0.1-10 \mathrm{~s}$ & Fractional Brownian motion \\
\hline & $(138)$ & 0.7 and 0.5 & $0.2-100 \mathrm{~s}$ & Rouse model \\
\hline \multirow{4}{*}{ 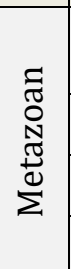 } & $(40)$ & ND & $0.01-15 \mathrm{~s}$ & $\begin{array}{l}\text { Two time regimes for "diffusive" } \\
\text { responses }\end{array}$ \\
\hline & $(130)$ & $0.3,0.5,1.2$ & $0.01-1000 \mathrm{~s}$ & Reptation \\
\hline & (139) & $0.4-0.7$ & $0.5-100 \mathrm{~s}$ & Subdiffusion \\
\hline & $(128)$ & 0.5 & $0.5-20 \mathrm{~s}$ & Subdiffusion \\
\hline
\end{tabular}

